\title{
The effect of animated consent material on participants' willingness to enrol in a placebo-controlled surgical trial: a protocol for a randomised feasibility study
}

\author{
Elizabeth Nelson ${ }^{1}$, Cade Shadbolt ${ }^{1}$, Samantha Bunzli ${ }^{1}$, Angela Cochrane ${ }^{2}$, Peter Choong ${ }^{1,2}$ and
} Michelle Dowsey ${ }^{1,2^{*}}$ (1)

\begin{abstract}
Background: Placebo-controlled surgical trials are recognised as the gold standard way to test the efficacy of a surgical procedure. Despite a rise in arthroscopic subacromial decompression (ASD) surgeries for the treatment of shoulder pain, only two placebo-controlled surgical trials have been conducted. These trials encountered significant recruitment challenges, threatening the external validity of findings. Difficulties with recruitment are common in clinical trials and likely to be amplified in placebo-controlled surgical trials. This mixed method feasibility trial aims to address the following questions: (i) Feasibility: What proportion of patients who have consented to undergo ASD report that they would be willing to enrol in a placebo-controlled trial for this procedure? (ii) Optimisation: Can patients' willingness to enrol in, or understanding of, such a trial be improved by supplementing written consent materials with a brief visual animation that outlines the details of the trial? And (iii) exploration: What factors influence patients stated willingness to enrol in such a trial, and how do they believe the recruitment process could be improved?
\end{abstract}

Methods: This study aims to recruit 80 patients on the waiting list for ASD. Participants will be randomised (1:1) to either view a brief video animation explaining the hypothetical placebo-controlled trial in addition to written information or to written information only. Participants in both groups will be required to state if they would be willing to opt-in to the hypothetical ASD trial after immediately being presented with the consent material and again 1 week after completion of the consent process. Patients in both groups will also be required to complete a measure of trial literacy. Twenty participants will be purposively sampled to take part in an embedded qualitative study exploring understanding of trial concepts and factors contributing to willingness to opt-in.

(Continued on next page)

\footnotetext{
* Correspondence: mmdowsey@unimelb.edu.au

${ }^{1}$ The University of Melbourne Department of Surgery, St. Vincent's Hospital, Level 2 Clinical Sciences Building, 29 Regent Street, Fitzroy, Melbourne, VIC 3065, Australia

${ }^{2}$ Department of Orthopaedics, St. Vincent's Hospital, Level 3 Daly Wing, 35 Victoria Parade, Fitzroy, Melbourne 3065, VIC, Australia
}

(c) The Author(s). 2021 Open Access This article is licensed under a Creative Commons Attribution 4.0 International License, which permits use, sharing, adaptation, distribution and reproduction in any medium or format, as long as you give appropriate credit to the original author(s) and the source, provide a link to the Creative Commons licence, and indicate if changes were made. The images or other third party material in this article are included in the article's Creative Commons licence, unless indicated otherwise in a credit line to the material. If material is not included in the article's Creative Commons licence and your intended use is not permitted by statutory regulation or exceeds the permitted use, you will need to obtain permission directly from the copyright holder. To view a copy of this licence, visit http://creativecommons.org/licenses/by/4.0/ The Creative Commons Public Domain Dedication waiver (http://creativecommons.org/publicdomain/zero/1.0/) applies to the data made available in this article, unless otherwise stated in a credit line to the data. 
(Continued from previous page)

Discussion: This feasibility study will provide evidence for optimising participant recruitment into a placebocontrolled trial of ASD by consenting patients using animated trial information in addition to written information. This pilot and feasibility data may also be relevant to placebo-controlled surgical trials more broadly, which are characterised by recruitment challenges.

Trial registration: ANZCTR, ACTRN12620001132932, date October 30, 2020

Keywords: Recruitment, Informed consent, Feasibility, Arthroscopic subacromial decompression, Orthopaedics, Placebo-controlled surgical trial, Sham surgery

\section{Background}

Placebo-controlled surgical trials are considered the gold standard for testing the efficacy of surgical procedures [1]. Although important disagreements about how best to define the concept of a surgical placebo have yet to be resolved [2], it is widely accepted that these control interventions should at the very least aim to mimic the standard surgery so patients are unaware of which arm of the trial they are enrolled in, and exclude any parts of the procedure that are thought to have a therapeutic effect $[1,3]$. By comparing the outcomes of patients who have been randomised to receive a placebo procedure to those who have had the standard surgery, trialists can assess the effectiveness of the standard therapy over and above non-specific and placebo effects. In orthopaedics, placebo-controlled surgical trials have helped change the practice landscape. The most well-known example of such changes relates to knee arthroscopy. Four placebocontrolled surgical trials have shown that knee arthroscopic lavage and debridement offer no benefit over and above the placebo effect [4-7]. Clinical guidelines now recommend against this procedure, suggesting that billions of dollars previously spent on these procedures every year should be put to better use $[8,9]$.

In light of these findings, placebo-controlled trials of other arthroscopic procedures are now garnering increased attention. One area of recent interest has been arthroscopic subacromial decompression (ASD) surgery for the treatment of shoulder pain [10, 11]. The two placebo-controlled trials of ASD conducted to date faced significant delays with recruitment. Comparing data from their clinical trial registry entries with their published results demonstrates that both trials ultimately required substantially more time to recruit than they had initially scheduled for both recruitment and patient follow-up. In both of these trials, the actual time to achieve target recruitment exceeded the target trial duration, with both trials requiring more than double the number of additional recruitment sites to achieve this. Difficulties with recruitment is common in clinical trials, with only one third meeting their target sample size [12]. This not only represents a significant waste of funds; it also raises ethical concerns. Patients who have enrolled in an underpowered or abandoned trial may have been exposed to unnecessary risk without contributing to the generation of new and meaningful medical knowledge [13]. The problem of low recruitment is likely to be amplified in placebo-controlled surgical trials. Patients may be reluctant to undergo an invasive placebo procedure without the expectation of some form of therapeutic benefit. Difficulties understanding concepts such as equipoise, placebo effects, randomisation, and blinding may also leave many patients confused and unwilling to participate in a placebocontrolled surgical trial [14].

Amongst the most commonly reported reasons for poor recruitment to surgical RCTs include difficulty understanding and a negative attitude towards trial concepts, and a preference for one form of treatment [14]. In line with these findings, Isaksson et al. [15] identified the two most important factors for enhancing recruitment in a RCT as (1) the research question needs to be considered important and (2) a simple procedure for providing information and gaining consent. One such strategy identified to help improve informed consent and therefore enhance recruitment includes the use of video animation. There is some evidence of the positive impact of video animation on enhancing patients' understandability of research and attitude about participation in clinical research [16-19]. While two studies have shown that the use of educational videos do not, on their own, have a substantial impact on patients' willingness to enrol in cancer-related trials $[16,17]$, the use of animated consent materials for placebo-controlled surgical trials has not been explored.

The challenges faced by previous trials highlight the pressing need to carefully evaluate the feasibility of placebo-controlled surgical trials early in the trial planning process. During the process of establishing the feasibility of such trials, there is also an imperative to assess the impact of concrete interventions that aim to optimise the trial's likelihood of success. Moreover, there is a need to explore new avenues to optimise the feasibility of planned trials by engaging with key stakeholders. Taken together, these considerations provide a 
framework for this mixed-methods feasibility study, which aims to address the following questions.

- Feasibility. What proportion of patients who have consented to undergo ASD report that they would be willing to enrol in a placebo-controlled trial for this procedure?

- Optimisation. Can patients' willingness to enrol in, or understanding of, such a trial be improved by supplementing written consent materials with a brief visual animation that outlines the details of the trial?

- Exploration. What factors influence patients stated willingness to enrol in such a trial, and how do they believe the recruitment process could be improved?

\section{Methods/design Trial design}

To answer each of the research question outlined above, we will undertake a two-arm, randomised feasibility trial with an embedded qualitative component. The quantitative component of this study has been designed to test the hypothesis that supplementing written consent materials with a brief animated video leads to patients being more likely to consent to a trial comparing a placebo-control to ASD. This study will be carried out at St. Vincent's Public Hospital Melbourne (SVHM) in Australia and will involve the recruitment of participant from both SVHM and from associated SVHM surgeon's private clinic lists. The study strategy is registered, constructed, and presented according to the recommendations of the SPIR IT [20] and CONSORT extension for pilot and feasibility trials guidelines [21] (see Additional File 1). Ethical approval has been obtained from St. Vincent's Hospital Melbourne Human Research Ethics Committee (reference number LRR 069/20, date 23 September, 2020). The trial has been registered with Australian New Zealand Clinical Trials Registry (ACTRN12620001132932, date October 30, 2020).

\section{Participants}

Given orthopaedic surgeons in Australia often work in both public and private settings, participants will be identified from the SVHM orthopaedic public clinic lists and from the private rooms of surgeons who conduct surgery at SVHM. Individuals will be consented to participate in the feasibility study via telephone call with a member of the research team. After approximately 1 week, individuals will be contacted again to confirm participation. Patient-specific health and sociodemographic information will be collected to test for relevant differences between patients in each arm of the trial. A set of three questions will be used to assess functional health literacy (adapted from Ghanouni et al. [22]). These questions will specifically measure participants stated ease of understanding of medical statistics and written information, and an objective numeracy measure. In addition, we will measure trial literacy using the Therapeutic Misconception Scale. A measure of the participants' general health status, including both physical and mental health, will be derived using the VR12 [23]. Symptom severity will be derived from a validated questionnaire (Oxford Shoulder Score [24]).

A subset of purposively sampled participants that represent a range of ages and gender from each study arm (approximately 20 in total) will be invited to participate in a phone audio-recorded qualitative interview. After the collection of all other outcome data, these participants will be asked to review the consent material (either written or video animation) while verbalising their immediate responses to these materials, using a think aloud' technique [25]. This will provide insight into how participants understand key concepts about the study design such as randomisation, blinding, and the use of placebo controls [26].

\section{Inclusion criteria}

Patients will be considered for inclusion if they are the following:

- Are aged 18 years or older

- Have consented for arthroscopic subacromial decompression (ASD) with one of the orthopaedic surgeons from St. Vincent's Hospital Melbourne (SVHM) or have consented to surgery at the private rooms of a surgeon who conducts surgery at SVHM

- Understand written and audio-visual instructions in English

\section{Exclusion criteria}

Patients will be excluded from consideration if they are the following:

- Undergoing revision surgery

- Unable to provide informed consent due to mental incompetence (e.g., intellectual disability, dementia)

- Non-English-speaking

\section{Planned interventions}

Participants will be presented with the informed consent materials for the arm to which they are randomised. Block randomisation will be used to allocate even numbers of participants to each arm. The consent materials used during the intervention will be presented to each participant individually, by one of the research team members. 


\section{Control}

This group will receive a written participant information and consent form based on a hypothetical trial, using the template for interventional studies mandated by the Victorian State Government. The content of the consent form has been informed by Australia's National Statement on Ethical Conduct in Human Research (NHMRC 2007) (see Additional File 2).

\section{Intervention}

In addition to the written participant information and consent form, participants randomised to the intervention arm will be provided with a 6-min-long video animation that explains a hypothetical trial in plain English. Content for the hypothetical trial is based on previously published placebo-controlled trials of ASD and informed by qualitative interviews with orthopaedic patients about their understanding of and attitude towards placebocontrolled surgical trials. The video animation will invite participants to take part in a hypothetical placebocontrolled trial of ASD. It will describe the procedure, the need for further research, what a placebo-controlled surgical trial involves, and trial participation requirements. It will also describe key concepts such as equipoise, placebo effects, randomisation, and blinding. All information represented in the consent video will also be contained in the written form.

\section{Outcomes}

\section{Primary outcome}

The primary outcome is the proportion of participants who state that they would be willing to opt into the hypothetical ASD trial after being presented with the relevant consent materials. As is common with complex trials, participants will be encouraged to review and discuss trial information before, during, and after consenting to participate. Therefore, outcome measures will be collected immediately after participating in the informed consent process $\left(t_{2}\right)$ and at a second time-point 1 week after completion of the consent process $\left(t_{3}\right)$.

\section{Secondary outcome}

The secondary outcome, trial literacy, will be assessed using the Therapeutic Misconception Scale [27]. Therapeutic misconception occurs when research participants fail to distinguish between the imperatives of clinical research and ordinary treatment. Therapeutic misconception is detrimental to a participant's understanding of a study, which is crucial for autonomous decision-making [27].

\section{Sample size justification}

As a feasibility study, no formal power calculation is needed [28]. Based on recommendations for sample sizes for feasibility studies [29], we have set a maximum target of 40 participants per arm (approximately 80 in total) to ensure adequate representation of males and females from both public and private hospital settings. In qualitative research, data collection and data analysis are conducted in parallel and the sample size is determined by thematic saturation. As such, an a priori sample size calculation is not appropriate as it is not possible to know in advance when saturation will be reached. Previous qualitative studies embedded in orthopaedic trials have reached saturation at 12 [30] to 18 participants [31]. Therefore, the sample size of 80 participants will also be sufficient to enable the embedded qualitative evaluation.

\section{Randomisation and masking}

To minimise bias, participants will be informed that the study aim is to explore patient understanding of informed consent procedures for a placebo surgery trial, but specific details about differences between these consent procedures will not be provided. Participants will only receive information on the mock trial arm they are randomised to and will receive no information about the comparator arm for the duration of the study. After being consented to this feasibility trial, participants will be randomly assigned in a ratio of 1:1 to either arm of the trial. Block randomisation will be performed by an investigator not involved in participant recruitment using computer-generated random assignment sequence prepared in blocks of four and stored in a passwordprotected file. A research assistant independent of recruitment and data collection will be responsible for participant management. The research associate (who will be responsible for patient consent) will be blinded to group allocation at the time of consent. In addition, outcome ascertainment will be blinded. Upon completion of the study, a biostatistician blinded to group allocation will analyse outcome data.

\section{Timelines}

The anticipated start date of November 2020 and end date of December 2021. A summary for SPIRIT schedule of study including enrolment, intervention, and assessment is shown in Table 1.

- Patients will receive a participant information form (via email) and given a verbal explanation of the project via telephone. Patients will be consented to be a part of this trial using a verbal consent script, which will be audio-recorded and documented (see Additional file 2).

- At this point, patient-specific health and sociodemographic information will be collected.

- Once participants have agreed to be a part of the study, they will be randomly assigned to view one 
Table 1 SPIRIT Trial study schedule of enrolment, interventions, and assessments

\begin{tabular}{|c|c|c|c|c|c|c|}
\hline \multirow[t]{2}{*}{ Time-point } & \multirow{2}{*}{$\begin{array}{l}\text { Enrolment } \\
-t_{1}\end{array}$} & \multirow{2}{*}{$\begin{array}{l}\text { Randomisation } \\
0\end{array}$} & \multicolumn{4}{|c|}{ Post-allocation } \\
\hline & & & $t_{1}$ & $t_{2}$ & $t_{3}$ & $t_{4}$ \\
\hline \multicolumn{7}{|l|}{ Enrolment } \\
\hline Baseline data, demographics & $x$ & & & & & \\
\hline Eligibility screen & $x$ & & & & & \\
\hline Informed consent & $x$ & & & & & \\
\hline Allocation & & $x$ & & & & \\
\hline \multicolumn{7}{|l|}{ Participant characteristics } \\
\hline Health literacy & $x$ & & & & & \\
\hline General Health $^{a}$ & $x$ & & & & & \\
\hline Symptom Severity ${ }^{b}$ & $x$ & & & & & \\
\hline \multicolumn{7}{|l|}{ Interventions } \\
\hline Video animation & & & $x$ & & & \\
\hline Written information & & & $x$ & & & \\
\hline \multicolumn{7}{|l|}{ Assessments } \\
\hline Recruitment rate & & & & $x$ & $x$ & \\
\hline Therapeutic misconception $^{c}$ & & & & $x$ & $x$ & \\
\hline \multicolumn{7}{|l|}{ Qualitative study } \\
\hline Think aloud interview & & & & & & $x$ \\
\hline
\end{tabular}

set of the consent materials for the hypothetical placebo-controlled ASD surgery trial.

- The main outcome measure will be participants stated willingness to opt-in to the hypothetical placebo-controlled shoulder surgery trial.

- Participants will also be asked a series of questions about their understanding of the information presented.

- This information will be collected at two timepoints: immediately after reviewing and discussing the trial information with a researcher, and again approximately 1 week after completion of the consent process: $2 \times 30$-min sessions.

- After the collection of outcome measures, a purposively sampled subset of participants will participate in qualitative interviews.

\section{Data management}

All data will be stored on a password-protected computer kept in a secure locked facility and only accessible to the research investigators and the trial coordinator as approved by the SVHM Human Research Ethics Committee (HREC). At the completion of the study, outcome data will be pooled and de-identified for analysis by a statistician. Due to the short duration and minimal risks of the trial, there will not be a data monitoring committee. However, the principal investigator will be responsible for overseeing the trial and ensuring data quality and completeness, including participant enrolment, consent eligibility and forms, allocation to study groups, data recording and timeliness of data collection. Furthermore, there will be no planned interim analyses and stopping guidelines.

\section{Analysis}

\section{Quantitative analysis}

Categorical variables will be analysed using chi-square tests. For continuous variables, we will employ (parametric) $t$ tests and (non-parametric) Mann-Whitney tests for symmetrically and asymmetrically distributed data, respectively. Analyses will be conducted on an intentionto-treat basis by a blinded statistician using Stata, version 14.0 (StataCorp, College Station, Texas).

\section{Qualitative analysis}

The qualitative interviews will gain further insight into how participants understand key study design concepts, and further the understanding of factors that contribute to participants' willingness to opt-in to the hypothetical trial. The interviews will be audio-recorded and transcribed data will be analysed using inductive thematic analysis [32] to compare data from participants randomised to written versus video animation informed consent. Concurrent with data collection, in the first stage 
of qualitative data analysis, two researchers (SB and EN) will independently identify concepts relevant to the research question in the first five interview transcripts. The two researchers will meet to discuss a preliminary coding framework, which will continue to be refined and extended through analysis of subsequent interviews. When the researchers are confident that the coding framework captures all relevant interview responses and no new codes are identified in subsequent interviews, recruitment will cease. Data will be uploaded into a qualitative data management software (Nvivo data management package, version 12.0) and all transcripts will be coded using the refined framework. Codes will be grouped thematically for each of the two study arms and themes between each study arm will be compared and contrasted. Emerging interpretations will be challenged through discussion amongst the inter-disciplinary research team.

\section{Discussion}

The proposed study will provide evidence that will inform the planning and design of future placebocontrolled surgical trials. Not only will it provide information about the proportion of patients that can be expected to enrol in such a trial, which is directly relevant to its overall feasibility, it will also provide insights into whether recruitment could be optimised through the use of supplemental animated consent materials. By embedding a qualitative investigation into this study, we hope to gain further insights into other barriers and anxieties faced by patients when considering participation in such a trial. This will provide important information for educating researchers involved in the recruitment process. Even in trials that are otherwise feasible, improving the patient recruitment process is likely to reduce both the time and funding needed to conduct the trial. Although these findings will be relevant to many forms of placebo-controlled surgical trials, they are likely to be most generalisable to trials of ASD.

More generally, this study will contribute to our understanding of whether the use of animated consent materials improves trial recruitment. While two studies have shown that the use of educational videos do not, on their own, have a substantial impact on patients' willingness to enrol in cancer-related trials (Du et al. 2008, 2009), the use of animated consent materials for placebo-controlled surgical trials has not been explored. Moreover, the only available study to assess the impact of providing a brief video in addition to written information showed that such supplemental information significantly increased willingness to enrol in a trial involving pregnant women with pre-labour rupture of membranes (Weston,
Hannah, and Downes 1997). Importantly, this trial does not simply hope to test whether such supplemental information is likely to improve recruitment, it will also assess the impact of such interventions on patients understanding of the trial to which they are consenting. Improved understanding of such trials allows patients to more appropriately express their autonomy, which is vital to the ethical conduct of clinical research.

There are potential limitations of the proposed study. Firstly, we will only include English-speaking participants. As this is a feasibility trial, an important first step is to validate the intervention in English before it is applied to a larger cohort and translated into other languages. Secondly, the authors acknowledge limitations associated with the hypothetical nature of the placebocontrolled surgical trial that participants are to consider joining [33]. This may result in an over-estimation of participants' willingness to opt-in. Consequently, recruitment rates reported in this trial will likely present an upper limit on the expected recruitment for a bona fide clinical trial in this domain. Importantly, the randomisation process should ensure that individuals' propensity to overstate their willingness to participate in the hypothetic trial is similar between groups. Consequently, the findings of this feasibility study will provide actionable evidence about the relative effect of offering patients animated trial information in addition to written information.

\section{Abbreviations}

ASD: Arthroscopic subacromial decompression; RCT: Randomised controlled trial; SVHM: St. Vincent's Public Hospital Melbourne; ANZCTR: Australian New Zealand Clinical Trials Registry; HREC: Human Research Ethics Committee

\section{Acknowledgements}

We would like to thank Margaret Fievez, a consumer representative, for her contribution towards the final development of the video animation. Michelle Dowsey holds a NHMRC Career Development Fellowship (1122526) and University of Melbourne Dame Kate Campbell Fellowship. Peter Choong holds a NHMRC Practitioner Fellowship (1154203). Cade Shadbolt is supported by the Australian Commonwealth Government through a Research Training Program Scholarship.

\section{Authors' contributions}

All authors contributed to the development of the study design and provided feedback on the protocol. EN and CS drafted the manuscript and all authors critically reviewed and revised the manuscript and approved the final version.

\section{Funding}

This project was supported by a University of Melbourne, Department of Surgery Seeding Grant. The funding source had no role in the study design and will not have any role during its conduct, collections, analyses, and interpretation of data or in the writing of reports and decision to submit for publication.

Availability of data and materials Not applicable 


\section{Ethics approval and consent to participate}

This study was approved by the St. Vincent's Hospital Human Research Ethics (reference number LRR 069/20) on 23 September 2020 and was registered with the Australian New Zealand Clinical Trials Registry (ANZCTR number ACTRN12620001132932) on 30 October 2020. The principal investigator will provide an annual progress report to the HREC, and the HREC will be notified in writing immediately of any proposed changes to the trial. Only once approval has been received from the HREC will changes be made to the trial and the clinical trial registry notified. Verbal consent will be obtained by the research associate from all study participants prior to enrolment. It is anticipated adverse events related to this trial will be few; however, if any do occur, they will be reviewed by the study chief investigator and reported to the HREC. Patients are free to withdraw from the trial at any time without providing a reason. Patients will be informed that if they decide to withdraw from the project, personal and health information already collected will be retained. Patients will be informed that if they do not want this to occur they are to notify the researcher at the time of withdrawing. Patients will also be informed that trial results will be shared with the scientific community and may be published in a scientific journal, but any such publication would not contain identifiable data. Details outlining participants' rights to access their data, study results, how study data will be used, and the processes in place to ensure individual confidentiality is protected, are all specified in the participant information and consent form (see Additional file 2).

\section{Consent for publication}

Not applicable

\section{Competing interests}

The authors declare no competing interests.

Received: 1 November 2020 Accepted: 26 January 2021

Published online: 08 February 2021

\section{Supplementary Information}

The online version contains supplementary material available at https://doi. org/10.1186/s40814-021-00782-7.

\section{Additional file 1:. CONSORT extension for Pilot and Feasibility Trials} Checklist

Additional file 2:. Verbal consent script and participant information form

Received: 1 November 2020 Accepted: 26 January 2021

Published online: 08 February 2021

\section{References}

1. Beard DJ, Campbell MK, Blazeby JM, Carr AJ, Weijer C, Cuthbertson BH, et al. Considerations and methods for placebo controls in surgical trials (ASPIRE guidelines). Lancet. 2020;395(10226):828-38.

2. Nunn R. It's time to put the placebo out of our misery. BMJ. 2009; 338(b1568).

3. Probst P, Grummich K, Harnoss JC, Huttner FJ, Jensen K, Braun S, et al. Placebo-controlled trials in surgery: a systematic review and meta-analysis. Medicine (Baltimore). 2016;95(17):e3516.

4. Bradley JD, Heilman DK, Katz BP, Gsell P, Wallick JE, Brandt KD. Tidal irrigation as treatment for knee osteoarthritis: a sham-controlled, randomized, double-blinded evaluation. Arthritis Rheum. 2002;46(1):100-8.

5. Moseley JB, O'Malley K, Petersen NJ, Menke TJ, Brody BA, Kuykendall DH, et al. A controlled trial of arthroscopic surgery for osteoarthritis of the knee. N Engl J Med. 2002;347(2):81-8.

6. Roos EM, Hare KB, Nielsen SM, Christensen R, Lohmander LS. Better outcome from arthroscopic partial meniscectomy than skin incisions only? A sham-controlled randomised trial in patients aged 35-55 years with knee pain and an MRI-verified meniscal tear. BMJ Open. 2018;8(2):e019461.

7. Sihvonen R, Paavola M, Malmivaara A, Itala A, Joukainen A, Nurmi H, et al. Arthroscopic partial meniscectomy versus sham surgery for a degenerative meniscal tear. N Engl J Med. 2013;369(26):2515-24.
8. National Institute for Health \& Care Excellence. Osteoarthritis: care \& management in adults. London; 2018.

9. Siemieniuk RAC, Harris IA, Agoritsas T, Poolman RW, Brignardello-Petersen R, Van de Velde $\mathrm{S}$, et al. Arthroscopic surgery for degenerative knee arthritis and meniscal tears: a clinical practice guideline. BMJ. 2017;357:j1982.

10. Beard DJ, Rees JL, Cook JA, Rombach I, Cooper C, Merritt N, et al. Arthroscopic subacromial decompression for subacromial shoulder pain (CSAW): a multicentre, pragmatic, parallel group, placebocontrolled, three-group, randomised surgical trial. Lancet. 2018; 391(10118):329-38.

11. Paavola M, Malmivaara A, Taimela S, Kanto K, Inkinen J, Kalske J, et al. Subacromial decompression versus diagnostic arthroscopy for shoulder impingement: randomised, placebo surgery controlled clinical trial. BMJ. 2018;362:k2860.

12. Bower P, Wallace P, Ward E, Graffy J, Miller J, Delaney B, et al. Improving recruitment to health research in primary care. Fam Pract. 2009;26(5):391-7.

13. Fogel DB. Factors associated with clinical trials that fail and opportunities for improving the likelihood of success: a review. Contemp Clin Trials Commun. 2018;11:156-64.

14. Abraham NS, Young JM, Solomon MJ. A systematic review of reasons for nonentry of eligible patients into surgical randomized controlled trials. Surgery. 2006;139(4):469-83.

15. Isaksson $E$, Wester $P$, Laska AC, Nasman $P$, Lundstrom E. Identifying important barriers to recruitment of patients in randomised clinical studies using a questionnaire for study personnel. Trials. 2019;20(1):618.

16. Du W, Mood D, Gadgeel S, Simon MS. An educational video to increase clinical trials enrollment among lung cancer patients. J Thorac Oncol. 2008; 3(1):23-9.

17. Du W, Mood D, Gadgeel S, Simon MS. An educational video to increase clinical trials enrollment among breast cancer patients. Breast Cancer Res Treat. 2009;117(2):339-47.

18. Hutchison C, Cowan C, McMahon T, Paul J. A randomised controlled study of an audiovisual patient information intervention on informed consent and recruitment to cancer clinical trials. Br J Cancer. 2007;97(6):705-11.

19. Strevel EL, Newman C, Pond GR, MacLean M, Siu LL. The impact of an educational DVD on cancer patients considering participation in a phase I clinical trial. Support Care Cancer. 2007;15(7):829-40.

20. Chan AW, Tetzlaff JM, Altman DG, Dickersin K, Moher D. SPIRIT 2013: new guidance for content of clinical trial protocols. Lancet. 2013;381(9861):91-2.

21. Eldridge SM, Chan CL, Campbell MJ, Bond CM, Hopewell S, Thabane L, et al. CONSORT 2010 statement: extension to randomised pilot and feasibility trials. BMJ. 2016;355:i5239.

22. Ghanouni A, Halligan S, Taylor SA, Boone D, Plumb A, Stoffel S, et al. Quantifying public preferences for different bowel preparation options prior to screening CT colonography: a discrete choice experiment. BMJ Open. 2014;4(4):e004327.

23. Kazis LE, Miller DR, Skinner KM, Lee A, Ren XS, Clark JA, et al. Applications of methodologies of the Veterans Health Study in the VA healthcare system: conclusions and summary. J Ambul Care Manage. 2006;29(2):182-8.

24. Dawson J, Fitzpatrick R, Carr A. Questionnaire on the perceptions of patients about shoulder surgery. J Bone Joint Surg Br. 1996;78(4):593-600.

25. Charters $E$. The use of think-aloud methods in qualitative research an introduction to think-aloud methods. Brock Education: a Journal of Educational Research and Practice. 2010;12(2).

26. Rooshenas L, Paramasivan S, Jepson M, Donovan JL. Intensive triangulation of qualitative research and quantitative data to improve recruitment to randomized trials: the QuinteT Approach. Qual Health Res. 2019:29(5):672-9.

27. Appelbaum PS, Anatchkova M, Albert K, Dunn LB, Lidz CW. Therapeutic misconception in research subjects: development and validation of a measure. Clin Trials. 2012;9(6):748-61.

28. Rahman U, Gemperle-Mannion E, Qureshi A, Edwin C, Smith TO, Parsons $\mathrm{H}$, et al. The feasibility of a randomised control trial to assess physiotherapy against surgery for recurrent patellar instability. Pilot Feasibility Stud. 2020;6:94

29. Sim J, Lewis M. The size of a pilot study for a clinical trial should be calculated in relation to considerations of precision and efficiency. J Clin Epidemiol. 2012;65(3):301-8.

30. Hinman RS, Nelligan RK, Bennell KL, Delany C. "Sounds a bit crazy, but it was almost more personal:" a qualitative study of patient and clinician 
experiences of physical therapist-prescribed exercise for knee osteoarthritis via Skype. Arthritis Care Res (Hoboken). 2017;69(12):1834-44.

31. Minns Lowe CJ, Moser J, Barker KL. Why participants in The United Kingdom Rotator Cuff Tear (UKUFF) trial did not remain in their allocated treatment arm: a qualitative study. Physiotherapy. 2018;104(2):224-31.

32. Braun F, Muller RU. Urinary extracellular vesicles as a source of biomarkers reflecting renal cellular biology in human disease. Methods Cell Biol. 2019; 154:43-65.

33. Treweek S, Lockhart P, Pitkethly M, Cook JA, Kjeldstrom M, Johansen $M$, et al. Methods to improve recruitment to randomised controlled trials: Cochrane systematic review and meta-analysis. BMJ Open. 2013; $3(2)$.

\section{Publisher's Note}

Springer Nature remains neutral with regard to jurisdictional claims in published maps and institutional affiliations.

Ready to submit your research? Choose BMC and benefit from:

- fast, convenient online submission

- thorough peer review by experienced researchers in your field

- rapid publication on acceptance

- support for research data, including large and complex data types

- gold Open Access which fosters wider collaboration and increased citations

- maximum visibility for your research: over $100 \mathrm{M}$ website views per year

At $B M C$, research is always in progress.

Learn more biomedcentral.com/submissions 